\title{
Propranolol inhibits infantile hemangioma by regulating the miR-424/vascular endothelial growth factor-A (VEGFA) axis
}

\author{
Zhen-Bo Wu ${ }^{1}$, Sen-Lin Shi ${ }^{1}$, Feng-Jun Pan ${ }^{2}, \mathrm{Li} \mathrm{Li}^{2}$, Hong-Yu Chen ${ }^{2}$ \\ ${ }^{1}$ College of Pharmacy, Zhejiang Chinese Medical University, Hangzhou, China; ${ }^{2}$ Department of Pharmacy, Lishui Municipal Central Hospital, \\ Lishui, China \\ Contributions: (I) Conception and design: ZB Wu, HY Chen; (II) Administrative support: L Li; (III) Provision of study materials or patients: ZB \\ Wu, SL Shi, HY Chen; (IV) Collection and assembly of data: All authors; (V) Data analysis and interpretation: ZB Wu, FJ Pan, HY Chen; (VI) \\ Manuscript writing: All authors; (VII) Final approval of manuscript: All authors. \\ Correspondence to: Hong-Yu Chen, Chief Pharmacist. Lishui Municipal Central Hospital, Lishui 323000, China. Email: chenhongyu0224@126.com.
}

Background: Infantile hemangioma (IHA) is the most common tumor in infancy. We aimed to explore the effect of propranolol on the expression of microRNA (miR)-424 in IHA tissues and XPTS-1 cells, as well as its molecular mechanism of inhibiting XPTS-1 cell activity.

Methods: Tumor tissues and peritumoral tissue were collected from 13 IHA patients in Lishui Municipal Central Hospital. The level of miR-424 were detected using real-time quantitative reverse transcription polymerase chain reaction (RT-PCR). Cell counting kit-8 (CCK-8) was used to measure XPTS-1 cell viability. Flow cytometry and transwell were used to detect the apoptosis level and invasion ability of XPTS-1 cells. Western blot was used to measure the protein level of vascular endothelial growth factor-A (VEGFA). The luciferase reporter gene assay detected the targeting relationship between miR-424 and VEGFA.

Results: Compared with normal tissues and human umbilical vein endothelial cells, the expression level of miR-424 in IHA tissues and XPTS-1 cells was significantly reduced $(\mathrm{P}<0.05)$. As the concentration of propranolol increased, XPTS-1 cell viability gradually decreased $(\mathrm{P}<0.05)$, and the expression level of VEGFA decreased $(\mathrm{P}<0.05)$. The expression of miR-424 increased with the time of propranolol treatment $(\mathrm{P}<0.05)$. Compared with the control group, treatment with an miR-424 inhibitor resulted in a significant increase in XPTS-1 cell viability and invasion ability $(\mathrm{P}<0.05)$, and a decrease in apoptosis $(\mathrm{P}<0.05)$. However, both propranolol and miR-424 inhibitor treatment resulted in a partial decrease in XPTS-1 cell viability $(\mathrm{P}<0.05)$, and a partial increase in the level of apoptosis $(\mathrm{P}<0.05)$. MiR-424 directly targeted VEGFA; the overexpression of miR-424 resulted in a decrease in the VEGFA protein level $(\mathrm{P}<0.05)$, while inhibition of miR-424 resulted in an increase in the VEGFA protein level $(\mathrm{P}<0.05)$. Compared with the propranolol group, the XPTS-1 cell viability and invasion ability in the propranolol + VEGFA-si group were significantly decreased $(\mathrm{P}<0.05)$, while the level of apoptosis increased $(\mathrm{P}<0.05)$. Meanwhile, simultaneous miR-424 inhibitor treatment resulted in no difference in cell viability and apoptosis levels compared with the propranolol group, and the invasion ability was partially restored $(\mathrm{P}<0.05)$.

Conclusions: Propranolol affects the malignant biological behavior of IHA cells by regulating the miR-424/VEGFA axis.

Keywords: Infantile hemangioma (IHA); propranolol; microRNA-424 (miR-424); vascular endothelial growth factor A (VEGFA)

Submitted May 10, 2021. Accepted for publication Jun 24, 2021.

doi: $10.21037 /$ tp-21-244

View this article at: https://dx.doi.org/10.21037/tp-21-244 


\section{Introduction}

Infantile hemangioma (IHA) is a benign tumor, which is characterized by irregular vascular structures formed by the abnormal proliferation of endothelial cells within 1 year of age (1). Although IHA is a benign tumor, this disease can cause facial deformation, breathing and vision obstruction in infants and young children, and can even be life-threatening (2). Therefore, it is necessary to study the related mechanisms of IHA and formulate reasonable treatment strategies.

Propranolol is currently the first-line treatment for IHA (3). The incidence of adverse reactions in children is relatively low, and it is a new option for the treatment of IHA. Pan et al. (4) reported that Pronell affects the expression of vascular endothelial growth factor (VEGF) by down-regulating the PI3K/Akt/eNOS pathway, thereby inhibiting the growth of the IHA endothelial cell line, XPTS-1. In addition, it inhibits the growth of IHA cells through $\beta 2$-adrenergic receptors in a HIF-1 $\alpha$-dependent manner (5). MicroRNA (miRNA) is a non-coding RNA with a length of approximately $22 \mathrm{bp}$, which plays an important role in numerous biological processes, such as cell proliferation, apoptosis, and tumor angiogenesis (6). Previous studies have confirmed that many miRNAs are involved in the development of IHA, such as miR-139, miR-130a, and miR-424 (7-9). As a tumor suppressor factor, miR-424 plays an important role in IHA. The Long non-coding RNA (lncRNA), metastasis associated in lung denocarcinoma transcript 1 (MALAT1), stimulates the MEKK3/NF- $\kappa$ B pathway by competitively binding miR-424 to promote the occurrence of IHA (10). Moreover, miR-424 regulates VEGFR-2 to inhibit the proliferation of IHA cells, and it also inhibits the phosphorylation of ERK1 and ERK2 proteins, inhibits cell proliferation, migration and blood vessel formation, regulates the development of IHA (11). However, it is not clear whether propranolol can inhibit the malignant biological behavior of IHA cells by up-regulating the expression of miR- 424 .

This study will analyze the expression of miR-424 in the tumor tissues of IHA patients and the IHA XPTS-1 cell line, and explore the molecules through which propranolol regulates the expression of miR-424 to affect the proliferation, apoptosis, and invasion of XPTS- 1 cells. This mechanism will provide new insights for exploring IHA treatment. We present the following article in accordance with the MDAR reporting checklist (available at https:// dx.doi.org/10.21037/tp-21-244).

\section{Methods}

\section{Material}

\section{Main reagents}

Dulbecco's modified eagle medium (DMEM) medium, Roswell Park Memorial Institute (RPMI) 1640 medium, and LipofectamineTM 2000 were purchased from Invitrogen, USA; fetal bovine serum, penicillin, streptomyces, trypsin, and Radio Immunoprecipitation Assay (RIPA) Lysis Buffer were purchased from Beijing Solabao Technology Co., Ltd. (Beijing, China); propranolol hydrochloride was purchased from Shanghai Latin Life Technology Co., Ltd. (Shanghai, China); TransZol Up Plus RNA Kit was purchased from Beijing Quanshijin Biotechnology Co., Ltd. (Beijing, China); PrimeScriptTM RT Master Mix was purchased from Tiangen Biochemical Technology (Beijing, China); Negative Control (NC) inhibitor, NC mimic, miR-424 inhibitor, and miR-424 mimic was purchased from Shanghai Abbots Biotechnology Co., Ltd. (Shanghai, China); primers were synthesized by Guangzhou Kinco Biotechnology Co., Ltd. (Guangzhou, China); TaqMan MicroRNA was purchased from Thermofisher, USA; specific primary antibodies [vascular endothelial growth factor-A (VEGFA) and glyceraldehyde-3phosphate dehydrogenase (GAPDH)] and secondary antibodies immunoglobulin-G (IgG) were purchased from Shanghai Abkang Trading Co., Ltd. (Shanghai, China); crystal violet, 4\% paraformaldehyde, Cell counting kit-8 (CCK-8) Kit, and Annexin V-FITC/PI Kit were purchased from Shanghai Biyuntian Biotechnology Co., Ltd.(Shanghai, China).

\section{Cell source}

According to the method of $\mathrm{Li}$ et al. (12), an immortalized human IHA-derived endothelial cell line, XPTS-1, was established. The specimen of this study was derived from an IHA patient (aged 2 months) who was admitted to our hospital. The IHA tissues were digested with trypsin and placed in DMEM medium for 16 subcultures, and then replaced with RPMI1640 medium to continue the culture. Human umbilical vein endothelial cells (HUVEC) were purchased from Wuxi Xinrun Biotechnology Co., Ltd. (Wuxi, China). The cells were cultured in RPMI1640 medium containing 10\% fetal bovine serum, $100 \mathrm{U} / \mathrm{mL}$ penicillin, and $100 \mathrm{mg} / \mathrm{mL}$ streptomycin. Both XPTS- 1 and HUVEC cells were cultured in an incubator at $37^{\circ} \mathrm{C}$ and $5 \%$ carbon dioxide $\left(\mathrm{CO}_{2}\right)$.

\section{Source of pathological tissue}

The tumor tissues of 13 IHA patients admitted to our hospital and the adjacent tissues $2 \mathrm{~mm}$ beyond the tumor tissue were 
collected. All procedures performed in this study involving human participants were in accordance with the Declaration of Helsinki (as revised in 2013). The study was approved by the Ethics Committee of Zhejiang Chinese Medical University \{NO.: Clinical Research Lun Shen [2021] No. [35]\} and informed consent was taken from all the patients.

\section{Experiment grouping}

The pathological tissues were divided into normal tissues and IHA tissues according to the pathological diagnosis; they were divided into HUVEC cell group and XPTS1 cell group according to the cells; and were divided into the following groups according to the experimental design: control group, no-load group, propranolol + no-load group, miR-424 inhibitor group (miR-424 inhibitor group), miR-424 overexpression group (miR-424 mimics group), propranolol + miR-424 inhibitor group, VEGF4 knockdown group (VEGF4-si group), wild-type VEGF4 group (VEGF4WT group), mutant VEGF4 group (VEGF4-MUT group), and propranel + VEGF4-si + miR-424 inhibitor group.

\section{Study method}

Real-time fluorescent quantitative polymerase chain reaction (PCR)

To detect miRNA expression, TransZol Up Plus RNA kit was used to extract the total RNA from tissues and cells. PrimeScriptTM RT Master Mix was used to reverse RNA into complementary DNA (cDNA), and TaqMan MicroRNA was then used to react in a fluorescent quantitative PCR machine (ABI7900, New York, USA). The procedure was as follows: pre-denaturation at $95^{\circ} \mathrm{C}$ for $2 \mathrm{~min}$, denaturation at $95^{\circ} \mathrm{C}$ for $15 \mathrm{~s}$, annealing at $60^{\circ} \mathrm{C}$ for $15 \mathrm{~s}$, extension at $72{ }^{\circ} \mathrm{C}$ for $20 \mathrm{~s}$, 40 amplification cycles, and extension at $72{ }^{\circ} \mathrm{C}$ for $15 \mathrm{~s}$. Primer: F-miR-424: 5'-GCGGCGGCAGCAGCAATTCATG-3', R-miR-424: 5'-ATCCAGTGCAGGGTCCGAGG-3'; F-U6: 5'-CTCGCTTCGGCAGCACA-3'，R-U6: 5'-AACGCTTCACGAATTTGCGT-3'. The $2^{-\Delta \Delta C t}$ method was used to calculate the relative expression of messenger RNA (mRNA).

\section{CCK-8 method to detect cell viability}

After the cells were treated in different ways, $5 \times 10^{3}$ cells were seeded in a 96 -well plate, $20 \mu \mathrm{L}$ of CCK- 8 solution was added, and the culture was continued for $4 \mathrm{~h}$. A microplate reader (Thermo Fisher Scientific, Massachusetts, USA) was used to measure the optical density at $490 \mathrm{~nm}$.

\section{Cell transfection}

After preparing XPTS-1 cells in the logarithmic growth phase into a cell suspension, approximately $1 \times 10^{5}$ cells were inoculated in each well of a six-well plate. The cells were placed in a constant temperature incubator at $37{ }^{\circ} \mathrm{C}$ and $5 \% \mathrm{CO}_{2}$ for 24 hours. According to the manufacturer's instructions, Lipofectamine ${ }^{\mathrm{TM}}$ 2000 was gently mixed with the plasmid containing the corresponding vector, let to stand, and then incubated at room temperature for $20 \mathrm{~min}$. Subsequently, the cell culture medium was added, mixed well, and allowed to culture for $24 \mathrm{~h}$. RT-PCR or Western blotting were used to detect transfection efficiency.

\section{Flow cytometry to detect apoptosis}

After the cells were treated in different ways, the apoptosis of XPTS-1 cells was detected by flow cytometry according to the annexin V-FITC/PI kit detection instructions. FlowJo software developed by Stanford University was used to analyze the level of apoptosis.

\section{Transwell method to detect cell invasion ability}

The cells of different treatments were prepared into cell suspensions and added to the upper chamber of the Transwell assay, and the lower chamber was filled with 10\% RPMI1640 culture medium. After allowing the cells to invade for 48 hours at room temperature, the cells in the upper chamber were removed with a cotton swab, and the cells in the lower chamber were fixed with $4 \%$ paraformaldehyde and stained with crystal violet. The cells were photographed and counted.

\section{Western Blotting detection of protein expression}

RIPA lysate was used to extract total protein from XPTS-1 cells. The protein was separated using $10 \%$ sulphate-polyacrylamide gel electrophoresis (SDS-PAGE), and then transferred to a polyvinylidene difluoride (PVDF) membrane. The membrane was washed in Tris Buffered Saline with Tween (TBST) containing $0.1 \%$ Tween-20 and blocked with 5\% skimmed milk. Next, the membrane was incubated with specific primary antibodies VEGFA $(1: 3,000)$ and GAPDH $(1: 3,000)$ at $4{ }^{\circ} \mathrm{C}$ overnight, and then with $\operatorname{IgG}(1: 10,000)$ in the dark at room temperature for $1 \mathrm{~h}$. Image J software e from National Institutes of Health was used to quantitatively analyze protein gray levels.

\section{Dual luciferase reporter gene experiment to detect the binding of the miRNA and target gene}

Lipofectamine $^{\mathrm{TM}} 2000$ was used to transfect the vector containing the mutation sites in the miR-186b and ABCG2 binding site sequence into the cells, and allowed to culture 
A

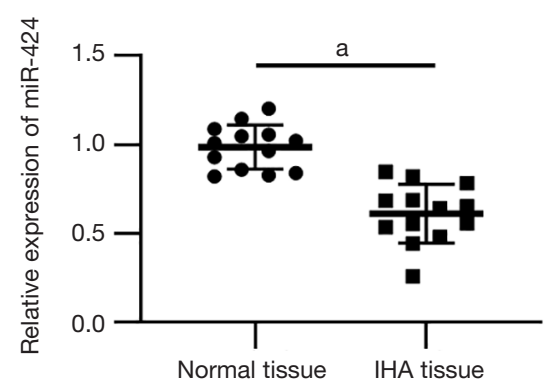

B

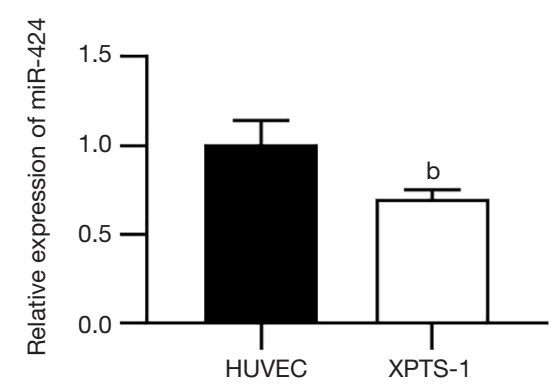

Figure 1 The expression of miR-424 in IHA tissues and cell lines. (A) The expression level of miR-424 in IHA tissue; (B) the expression level of miR-424 in IHA cell line; ${ }^{\text {a }}, \mathrm{P}<0.05$, compared with normal tissue; ${ }^{\text {b }}, \mathrm{P}<0.05$, compared with HUVECs. IHA, infantile hemangioma; HUVEC, human umbilical vein endothelial cell.

A

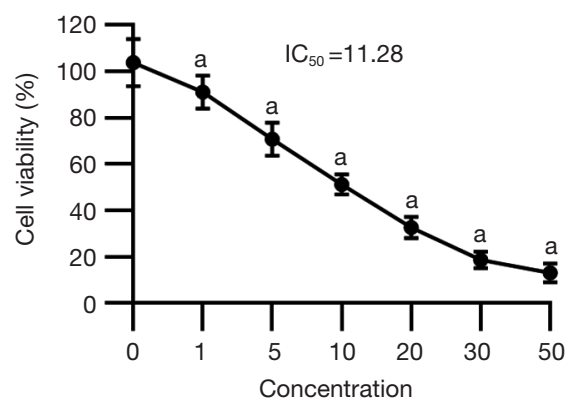

B

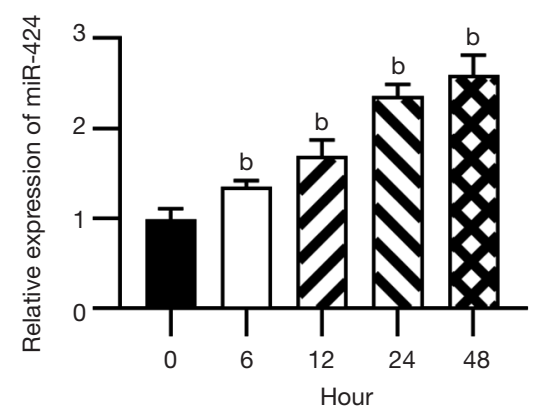

Figure 2 Effects of propranolol on the viability of IHA cells and the expression of miR-424. (A) The effect of propranolol on the viability of IHA cells; (B) the effect of propranolol on the expression of miR-424. ${ }^{\mathrm{a}}, \mathrm{P}<0.05$, compared with $0 \mathrm{nmol} / \mathrm{L} ;{ }^{\mathrm{b}}, \mathrm{P}<0.05$, compared with $0 \mathrm{~h}$. IHA, infantile hemangioma.

for 48 hours. The dual luciferase reporter gene kit was used for analysis according to the manufacturer's instructions, and luciferase activity was measured.

\section{Statistical analysis}

All data were statistically analyzed by SPSS 19.0 software which developed by IBM (Armonk, New York, USA), and experimental statistics were drawn by GraphPad Prism 8 software (GraphPad Software, Inc., California, US; Version 8). Normally distributed data between the two groups was analyzed by the Student's $t$-test. $\mathrm{P}<0.05$ was considered to indicate a statistically significant difference.

\section{Results}

\section{The expression of miR-424 in IHA tissues and cell lines}

RT-PCR was used to detect the expression level of miR-424 in IHA tissues and the XPTS-1 cell line. The results showed that the expression levels of miR-424 in IHA tissues and XPTS- 1 cells were significantly lower than those in normal tissues and HUVECs $(\mathrm{P}<0.05$, Figure 1).

\section{Effects of propranolol on the viability of IHA cells and the expression of $\mathrm{miR}-424$}

The inhibitory effect of propranolol on IHA cell viability was detected using the CCK-8 method. The results showed that as the concentration of propranolol increased, the viability of IHA cells gradually decreased $(\mathrm{P}<0.05$, Figure $2 A$ ), and the half-limiting dose (IC50) was $11.28 \mathrm{nmol} / \mathrm{L}$. Therefore, in the subsequent experiments, a concentration of $10 \mathrm{nmol} / \mathrm{L}$ was used to treat the cells. The effect of propranolol on the expression of miR-424 was detected by RT-PCR, and the results showed that the expression level of miR-424 increased with the time of propranolol treatment $(\mathrm{P}<0.05$, Figure 2B). 
A

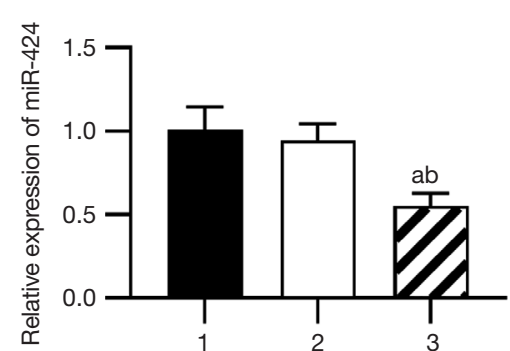

C

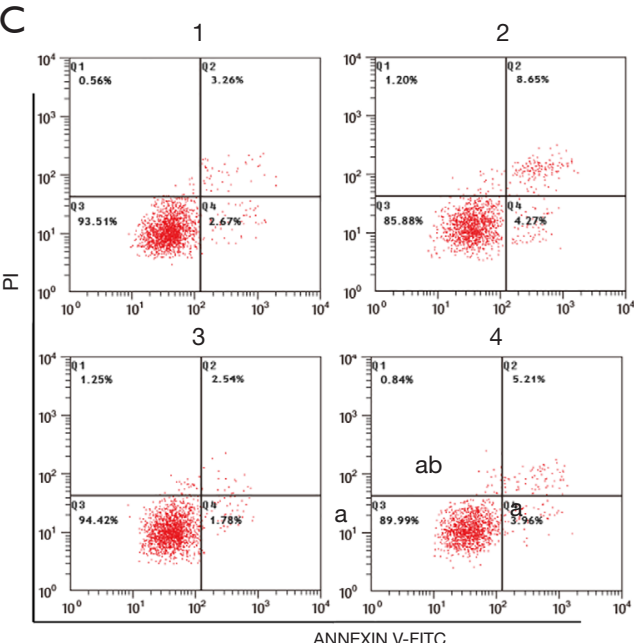

D

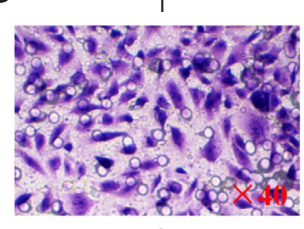

3

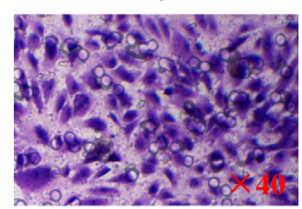

B
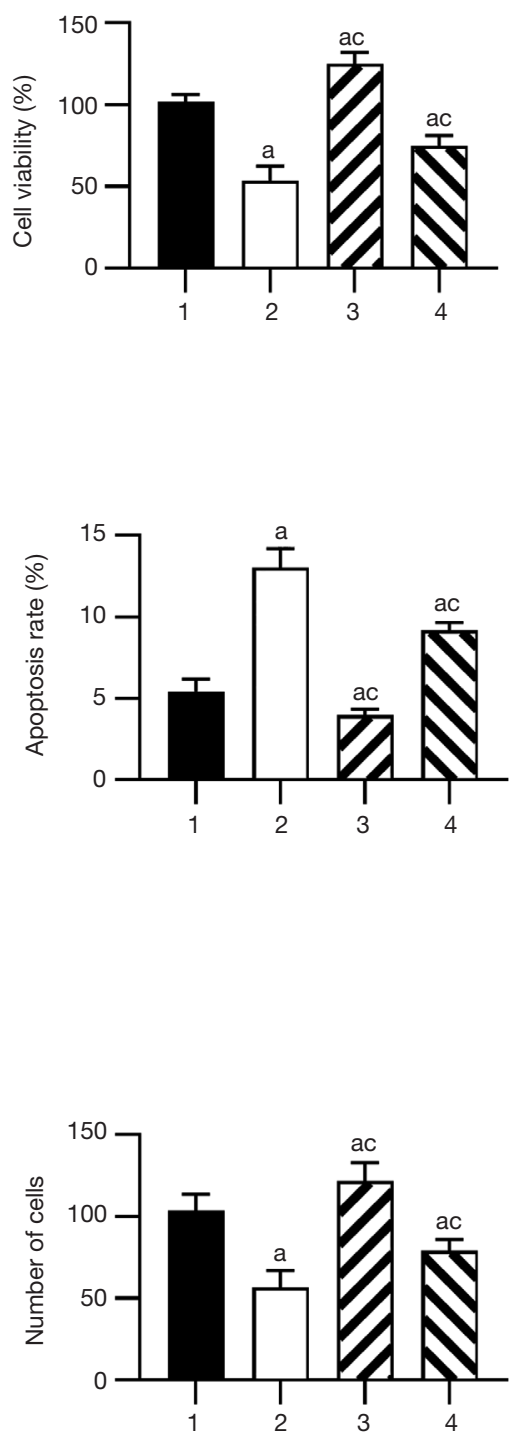

Figure 3 Propranolol inhibits IHA cell activity by regulating miR-424. (A) Transfection efficiency of the miR-424 inhibitor (1: control group; 2: no-load group; 3: miR-424 inhibitor group); (B) cell viability (1: control group; 2: propranolol group; 3: miR-424 inhibitor group; 4: propranolol + miR-424 inhibitor group); (C) apoptosis rate (1: control group; 2: propranolol group; 3: miR-424 inhibitor group; 4: propranolol + miR-424 inhibitor group); (D) cell invasion ability (1: control group; 2: propranolol group; 3: miR-424 inhibitor group; 4: propranolol + miR-424 inhibitor group. cells were stained with $0.5 \%$ crystal violet solution; magnification, $40 \times)$. ${ }^{\text {a }}, \mathrm{P}<0.05$, compared with the control group; b, $\mathrm{P}<0.05$, compared with the no-load group; ${ }^{\mathrm{c}}, \mathrm{P}<0.05$, compared with the propranolol group. IHA, infantile hemangioma.

\section{Propranolol inhibits IHA cell activity by regulating miR-424}

Compared with the control and no-load groups, the expression level of miR-424 in the miR-424 inhibitor group was significantly reduced $(\mathrm{P}<0.05$, Figure $3 A)$. Compared with the control group, propranolol treatment reduced XPTS-1 cell viability and invasion ability $(\mathrm{P}<0.05)$, while the apoptosis level increased $(\mathrm{P}<0.05)$. Also, inhibiting the expression of miR-424 increased cell viability and significantly increased the invasion ability $(\mathrm{P}<0.05)$, while the level of apoptosis decreased $(\mathrm{P}<0.05)$. Compared with the propranolol group, simultaneous treatment with propranolol and the miR424 inhibitor resulted in partial restoration of cell viability, invasion ability, and apoptosis level $(\mathrm{P}<0.05$, Figure $3 B, C, D)$. 
A

VEGFA 5'-cauuuuauuuucUUGCUGCUa-3'

IIIIIIII

miR-424 3'-aaguuuuguacuuAACGACGAC-5'

C

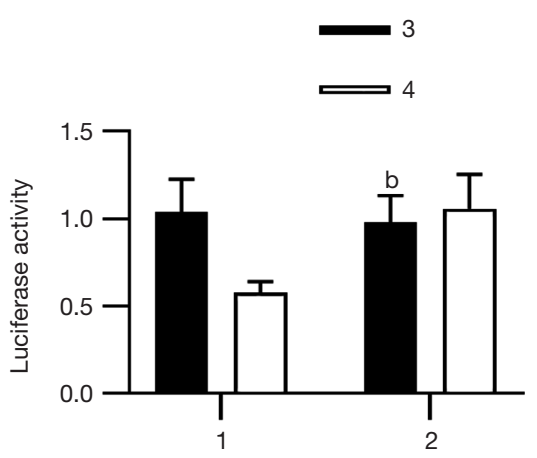

B

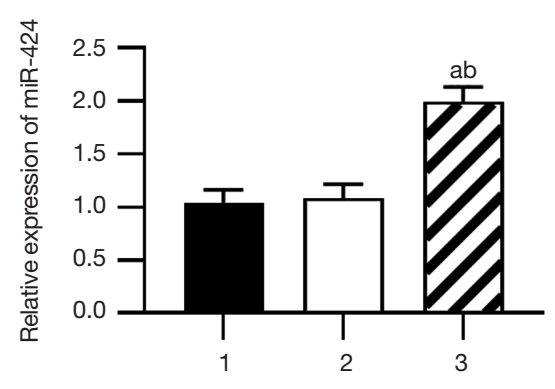

D
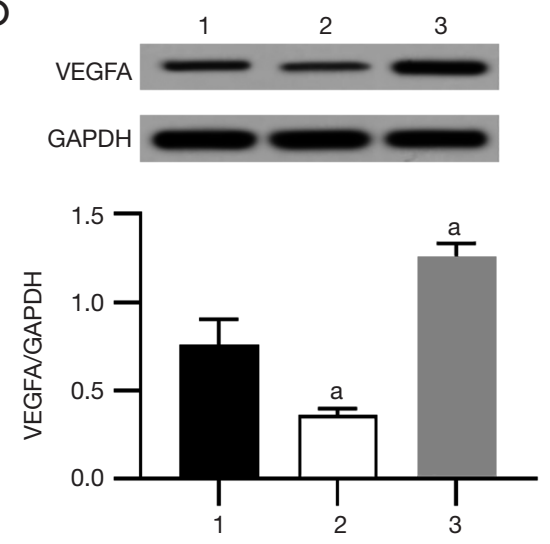

Figure 4 miR-424 targets and regulates VEGFA. (A) Binding sites of miR-424 and VEGFA; (B) transfection efficiency of miR-424 mimics (1: control group; 2: empty group; 3: miR-424 mimics group); (C) relative luciferase activity (1: VEGF4-WT group; 2: VEGF4-MUT group; 3: no-load group; 4: miR-424 mimics group); (D) effect of miR-424 on VEGFA expression (1: control group; 2: empty carrier group; 3: miR424 inhibitor group). ${ }^{\text {, }}, \mathrm{P}<0.05$, compared with the control group; ${ }^{\mathrm{b}}, \mathrm{P}<0.05$, compared with the no-load group. VEGFA, vascular endothelial growth factor-A; VEGF4-WT group, wild-type VEGF4 group; VEGF4-MUT group, wild-type VEGF4 group.

\section{miR-424 targets and regulates VEGFA}

The targeted binding sites of miR-424 and VEGFA were predicted through the starBase online website (https:// www.starbasegame.com/) (Figure 4A). First, RT-PCR was used to detect the transfection efficiency of the miR-424 mimic. Compared with the control and no-load groups, the expression level of miR-424 in the miR-424 mimic group was significantly up-regulated $(\mathrm{P}<0.05$, Figure $4 B)$. We also found that transfection of the miR-424 mimic resulted in a significant decrease in luciferase activity in the VEGFAWT group compared to the NC mimic $(\mathrm{P}<0.05$, Figure $4 C)$, further verifying the targeted regulation relationship between miR-424 and VEGFA. In the VEGFA-MUT group, the NC mimic and miR-424 mimic treatments did not affect the luciferase activity. Finally, the effect of miR424 on the expression of VEGFA was detected by western blot. Compared with the control group, transfection of the
miR-424 mimic resulted in a significant decrease in the protein level of VEGFA $(\mathrm{P}<0.05)$, while transfection of the miR-424 inhibitor resulted in a significant increase in the level of VEGFA $(\mathrm{P}<0.05$, Figure $4 D)$.

\section{Propranolol affects IHA cell activity via the miR-424/ VEGFA axis}

Firstly, the effect of different concentrations of propranolol on the expression of VEGFA was tested, and the results showed that the expression level of VEGFA decreased with propranolol in a concentration-dependent manner $(\mathrm{P}<0.05$, Figure 5). Furthermore, the molecular mechanism of propranolol in the inhibition of IHA was also explored. Compared to the control group, propranolol treatment significantly reduced the protein level of VEGFA $(\mathrm{P}<0.05$, Figure $6 A$ ). In order to test whether propranolol affects the viability of IHA cells via miR-424/VEGFA, a VEGFA-si 

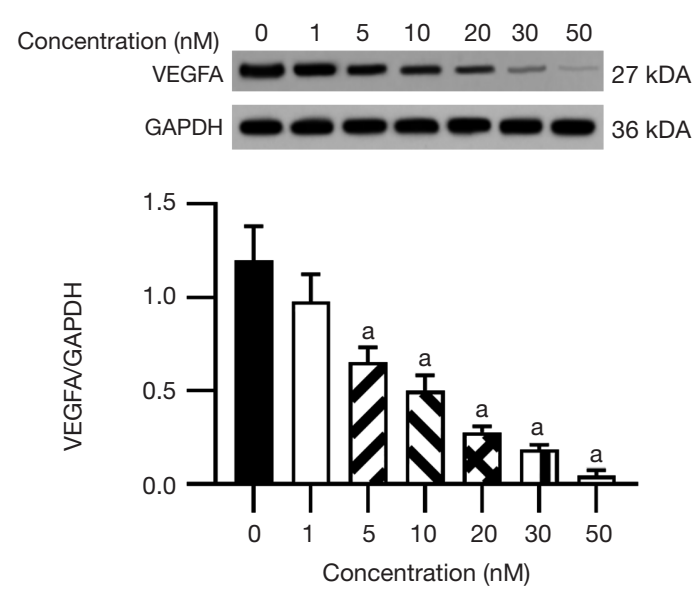

Figure 5 The effect of propranolol on the expression of VEGFA. a, $\mathrm{P}<0.05$, compared with $0 \mathrm{nmol} / \mathrm{L}$ group. VEGFA, vascular endothelial growth factor-A.

vector was constructed. Compared to the control and noload groups, the VEGFA protein level in the VEGFA-si group was significantly reduced $(\mathrm{P}<0.05$, Figure $6 A)$. We also found that, compared to the propranolol group, propranolol + VEGFA-si treatment resulted in a further decrease in cell viability and invasion ability $(\mathrm{P}<0.05)$, while the level of apoptosis increased $(\mathrm{P}<0.05$, Figure $6 B, C, D)$. Simultaneous treatment with nadolol + VEGFA-si + miR-424 inhibitor resulted in the recovery of cell viability and apoptosis levels that were not significantly different from those of the propranolol group, while the invasion ability was slightly lower than that of the propranolol treatment group $(\mathrm{P}<0.05)$.

\section{Discussion}

As the first-line drug for IHA, propranolol has the advantages of being low cost and having few side effects. Although it has been widely used in the clinical treatment of IHA and its basic pharmacological mechanism has been elucidated, the deeper molecular mechanism remains inconclusive. Therefore, there is a pressing need to understand the molecular mechanism of propranolol in the treatment of IHA, which will contribute to the development of new therapies for IHA.

This study aimed to explore the molecular mechanism of miRNA involved in the treatment of IHA with propranolol. MiRNAs are involved in the development and disorders of hemangioma. For example, miR-29a, miR-206, and miR-455 participate in a variety of biological functions at different stages of IHA (13). The role of miR-424 in IHA has also been widely reported. Yang et al. (14) found that miR-424 can inhibit the proliferation, migration, and angiogenesis of IHA cells by inhibiting the basic fibroblast growth factor/Fibroblast Growth Factor Receptor 1 (bFGF/ FGFR1) pathway to enhance extracellular regulated protein kinases (ERK1/2) phosphorylation. Our results are similar to those of previous studies. The expression of miR-424 in IHA tissues and cell lines was significantly reduced. In addition, the present study also found that its expression level increased with the increase of processing time. This indicates that propranolol may up-regulate the expression of miR-424 to inhibit IHA. Numerous studies have shown that propranolol can play a role by regulating the expression of miRNA. For example, propranolol inhibits the proliferation of IHA stem cells and promotes apoptosis by up-regulating the expression of miR-187-3p and miR-125b $(15,16)$. In this study, propranolol regulated the viability, invasiveness, and apoptosis of XPTS-1 cells by up-regulating the expression of miR-424. When treated with propranolol and the miR-424 inhibitor at the same time, the malignant biological behavior mediated by the miR-424 inhibitor was partially inhibited, which shows that miR-424 is sensitive to propranolol and plays an important role in monitoring the therapeutic effect of propranolol.

VEGF receptor signaling plays a vital role in regulating the biological behavior of endothelial cells derived from hemangioma (17). There are six members in the VEGF family, including VEGFA, VEGFB, VEGFC, VEGFD, VEGFE, and placental growth factor (PIGF). Compared with the other members, VEGFA is considered to be the most important molecular target for the treatment of IHA $(18,19)$. It has been reported that the single nucleotide polymorphism of the VEGFA gene is a risk factor for increasing IHA (20). In addition, some studies have pointed out that propranolol affects the development of IHA by regulating the expression of VEGFA $(4,5)$. The results of this study show that propranolol can inhibit the expression of VEGFA by regulating miR-424. In the early stage, the downstream target genes of miR-424 were predicted using starBase, and it was found that VEGFA and miR-424 had targeted binding sites. The dual luciferase reporter gene experiment verified their targeting relationship. Functional studies have found that, compared with propranolol treatment alone, simultaneous treatment with propranolol and VEGFA-si resulted in significantly reduced XPTS-1 cell viability and invasion ability. After adding the miR-424 inhibitor, XPTS-1 cell viability, apoptosis level, and invasion 
A
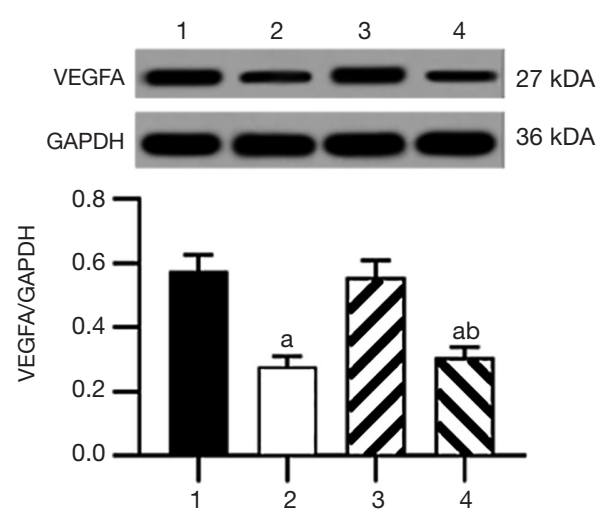

B

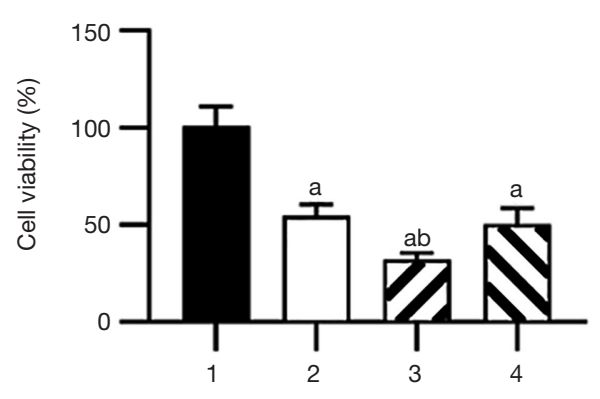

C
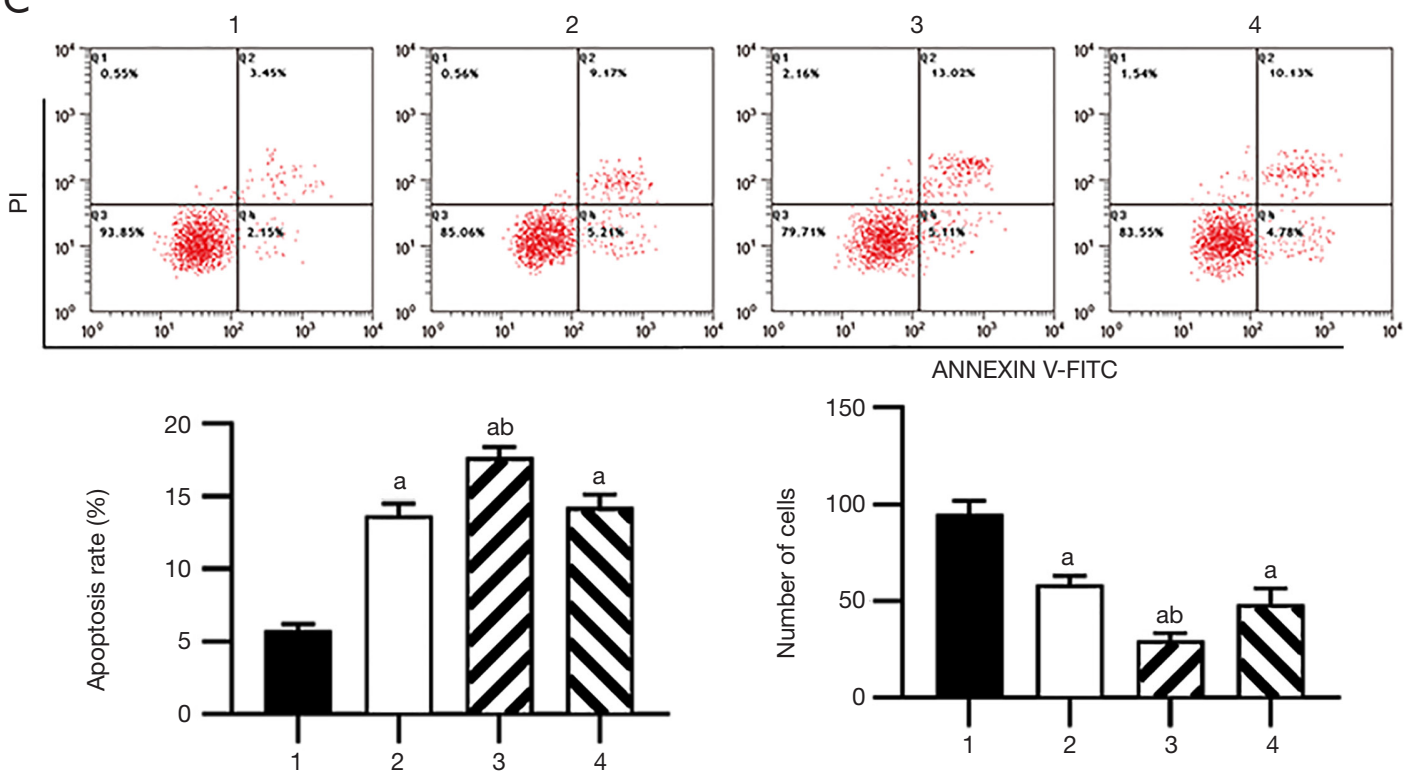

D
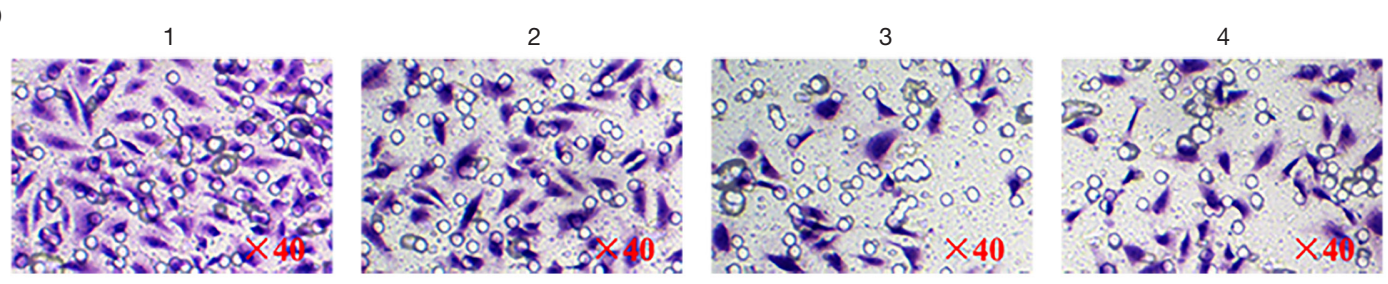

Figure 6 Propranolol affects IHA cell activity via the miR-424/VEGFA axis. (A) VEGFA-si transfection efficiency (1: control group; 2: propranolol group; 3: empty group; 4: VEGFA-si group); (B) cell viability (1: control group; 2: general narolol group; 3: propranolol + no-load group; 4: propranolol + VEGFA-si + miR-424 inhibitor group); (C) apoptosis rate (1: control group; 2: propranolol group); 3: propranolol + no-load group; 4: propranolol + VEGFA-si + miR-424 inhibitor group); (D) cell invasion ability (1: control group; 2: propranolol Group; 3: propranolol + no-load group; 4: propranolol + VEGFA-si + miR-424 inhibitor group. cells were stained with $0.5 \%$ crystal violet solution; magnification, $40 \times$ ). a, $\mathrm{P}<0.05$, compared with the control group; ${ }^{\text {b }}, \mathrm{P}<0.05$, compared with the propranolol group. IHA, infantile hemangioma; VEGFA, vascular endothelial growth factor-A.

ability were partially restored. These results indicate that propranolol can affect the malignant biological behavior of IHA cells through the miR-424/VEGFA axis. In this study, the angiogenesis ability of XPTS-1 cells was not investigate; however, previous studies have reported that miR-424 is also involved in the regulation of angiogenesis, mediates peroxisome proliferator-activated receptor $\gamma(\operatorname{PPAR} \gamma)$, and participates in inflammation-mediated angiogenesis (21). In 
dental pulp cells, miR-424 has also demonstrated an ability to inhibit angiogenesis (22). Therefore, it is speculated that miR-424 can also affect the angiogenesis process through VEGFA in IHA. This will be studied in more depth in future research.

Overall, this in vitro study confirmed that propranolol can regulate miR-424 and thus affect IHA. This process may inhibit the proliferation and invasion of IHA cells and induce apoptosis by targeting VEGFA, indicating that propranolol can be used in the treatment of IHA. This study provides insights for the development of new treatment methods for IHA.

\section{Acknowledgments}

Funding: None.

\section{Footnote}

Reporting Checklist: The authors have completed the MDAR reporting checklist. Available at https://dx.doi. org/10.21037/tp-21-244

Data Sharing Statement: Available at https://dx.doi. org/10.21037/tp-21-244

Conflicts of Interest: All authors have completed the ICMJE uniform disclosure form (available at https://dx.doi. org/10.21037/tp-21-244). The authors have no conflicts of interest to declare.

Ethical Statement: The authors are accountable for all aspects of the work in ensuring that questions related to the accuracy or integrity of any part of the work are appropriately investigated and resolved. All procedures performed in this study involving human participants were in accordance with the Declaration of Helsinki (as revised in 2013). The study was approved by the Ethics Committee of Zhejiang Chinese Medical University [NO.: Clinical Research Lun Shen (2021) No. (35)] and informed consent was taken from all the patients.

Open Access Statement: This is an Open Access article distributed in accordance with the Creative Commons Attribution-NonCommercial-NoDerivs 4.0 International License (CC BY-NC-ND 4.0), which permits the noncommercial replication and distribution of the article with the strict proviso that no changes or edits are made and the original work is properly cited (including links to both the formal publication through the relevant DOI and the license). See: https://creativecommons.org/licenses/by-nc-nd/4.0/.

\section{References}

1. Léauté-Labrèze C, Harper JI, Hoeger PH. Infantile haemangioma. Lancet 2017;390:85-94.

2. Chen ZY, Wang QN, Zhu YH, et al. Progress in the treatment of infantile hemangioma. Ann Transl Med 2019;7:692.

3. Chang L, Gu Y, Yu Z, et al. When to stop propranolol for infantile hemangioma. Sci Rep 2017;7:43292.

4. Pan WK, Li P, Guo ZT, et al. Propranolol induces regression of hemangioma cells via the down-regulation of the PI3K/Akt/eNOS/VEGF pathway. Pediatr Blood Cancer 2015;62:1414-20.

5. Li P, Guo Z, Gao Y, et al. Propranolol represses infantile hemangioma cell growth through the $\beta 2$-adrenergic receptor in a HIF- $1 \alpha$-dependent manner. Oncol Rep 2015;33:3099-107.

6. Shen Z, Gu X, Mao W, et al. Characterization of microRNA expression profiles by deep sequencing of small RNA libraries in leukemia patients from Naxi ethnic. Transl Cancer Res 2019;8:160-9.

7. Wang SJ, Li YJ, Gao B, et al. Long non-coding RNA 00152 slicing represses the growth and aggressiveness of hemangioma cell by modulating miR-139-5p. Biomed Pharmacother 2019;120:109385.

8. Gao F, Wang FG, Liu RR, et al. Epigenetic silencing of miR-130a ameliorates hemangioma by targeting tissue factor pathway inhibitor 2 through FAK/PI3K/Rac1/ mdm2 signaling. Int J Oncol 2017;50:1821-31.

9. Yang L, Dai J, Li F, et al. The expression and function of miR-424 in infantile skin hemangioma and its mechanism. Sci Rep 2017;7:11846.

10. Li MM, Dong CX, Sun B, et al. LncRNA-MALAT1 promotes tumorogenesis of infantile hemangioma by competitively binding miR-424 to stimulate MEKK3/NFкB pathway. Life Sci 2019;239:116946.

11. Fei Z, Qiu M, Qi X, et al. MicroRNA-424 suppresses the proliferation of hemangioma-derived endothelial cells by targeting VEGFR-2. Mol Med Rep 2018;18:4065-71.

12. Li P, Xiao XE, Xu Q, et al. Establishment of human infancy hemangioma-derived endothelial cell line XPTS1 and animal model of human infancy hemangioma. Zhonghua Kou Qiang Yi Xue Za Zhi 2011;46:129-33.

13. Chen J, Li C, Li Y, et al. Mechanisms of Action of MicroRNAs in Infantile Hemangioma Tissue and Vascular 
Endothelial Cells in Different Periods. Med Sci Monit 2017;23:4214-24.

14. Yang L, Dai J, Li F, et al. The expression and function of miR-424 in infantile skin hemangioma and its mechanism. Sci Rep 2017;7:11846.

15. Liu C, Zhao Z, Ji Z, et al. MiR-187-3p Enhances Propranolol Sensitivity of Hemangioma Stem Cells. Cell Struct Funct 2019;44:41-50.

16. Huang J, Jiang D, Zhao S, et al. Propranolol suppresses infantile hemangioma cell proliferation and promotes apoptosis by upregulating miR-125b expression. Anticancer Drugs 2019;30:501-7.

17. Huang C, Huang J, Yu G. Co-suppression of VEGF-A and VEGF-C inhibits development of experimental hemangioma. Am J Transl Res 2018;10:2911-9.

18. Greenberger S, Adini I, Boscolo E, et al. Targeting NF$\kappa \mathrm{B}$ in infantile hemangioma-derived stem cells reduces

Cite this article as: $\mathrm{Wu} Z \mathrm{ZB}$, Shi SL, Pan FJ, Li L, Chen HY. Propranolol inhibits infantile hemangioma by regulating the miR-424/vascular endothelial growth factor-A (VEGFA) axis. Transl Pediatr 2021;10(7):1867-1876. doi: 10.21037/tp-21-244
VEGF-A expression. Angiogenesis 2010;13:327-35.

19. Greenberger S, Boscolo E, Adini I, Mulliken JB, Bischoff J. Corticosteroid suppression of VEGF-A in infantile hemangioma-derived stem cells. N Engl J Med 2010;362:1005-13.

20. Oszajca K, Szemraj J, Wyrzykowski D, et al. Singlenucleotide polymorphisms of VEGF-A and VEGFR-2 genes and risk of infantile hemangioma. Int J Dermatol 2018;57:1201-7.

21. Lee A, Papangeli I, Park Y, et al. A PPAR $\gamma$-dependent miR-424/503-CD40 axis regulates inflammation mediated angiogenesis. Sci Rep 2017;7:2528.

22. Liu W, Gong Q, Ling J, et al. Role of miR-424 on angiogenic potential in human dental pulp cells. J Endod 2014;40:76-82.

(English Language Editor: A. Kassem) 